\title{
FLUID FLOW AND HEAT TRANSFER OF NATURAL CONVECTION ADJACENT TO UPWARD-FACING, INCLINED, HEATED PLATE (AIR CASE)
}

\author{
Fumiyoshi KIMURA* and Kenzo KITAMURA**
}

\author{
*Dept. of Mech. and Sys. Eng., Univ. of Hyogo, 2167, Shosha, Himeji, Hyogo 671-2280, Japan \\ ** Dept. of Mech. Eng., Toyohashi Univ. of Tech., Tempaku-cho, Toyohashi, Aichi 441-8580, Japan
}

\begin{abstract}
Experimental investigation have been performed on natural convection of air over an upward facing, inclined, heated plate. The special interests were paid to the turbulent transition and the heat transfer characteristics. The flow fields over the plate were visualized with smoke. The results showed that longitudinal vortices appear first in the laminar boundary layer when $\theta<72^{\circ}$. Then, the vortices detach from the plate and become distorted, and, finally, a fully turbulent state is accomplished at far downstream of the plate. Local Nusselt numbers from the plate were also measured. The results showed that the numbers deviate from the laminar values with the formation of the longitudinal vortices and, then, increase significantly with the detachment of the vortices. Based on these results, empirical correlations for the laminar and turbulent heat transfer by natural convection were proposed.
\end{abstract}

Keywords: Convection, Heat Transfer, Inclined Plate, Turbulent Transition, Solar Thermal Energy Utilization.

\section{INTRODUCTION}

Natural convective flows induced over upward-facing inclined heated plates are encountered in a wide variety of industrial equipment and of environmental situations. In particular, in the field of solar thermal energy utilizations, the knowledge on the flow and heat transfer of the natural convections of air is indispensable to the design of solar collectors and receivers. Therefore, intensive studies have been carried out to investigate their flow and heat transfer characteristics both experimentally and analytically.

Reviewing previous investigations, the main concerns seem to be directed to the following two groups of problems. One encompasses the problems concerned with the heat transfer from the inclined plate. The other encompasses the problems concerned with the mechanisms of turbulent transition. Thus, we will mention the relevant works with this order. Hassan and Mohamed ${ }^{[1]}$ have measured the local heat transfer coefficients from the isothermal plate to the laminar flows of air, where the inclination angle of the plate was varied as $\theta=15-90^{\circ}$ from horizontal. They showed that the laminar Nusselt numbers, $N u_{x}\left(=h_{x} \cdot x / \lambda\right)$, are correlated well with the local Rayleigh numbers, $R a_{x \theta}\left(=g \sin \theta \cdot \beta \Delta T x^{3} / \alpha v\right)$, whose gravitational acceleration was adopted as $g \sin \theta$ instead of $g$ itself, and that the data plotted on the $N u_{x}-R a_{x \theta}$ plane coincide fairly well the conventional correlation for the vertical plate. Chen and his colleagues ${ }^{[2]}$ have carried out a similarity analysis on the heat transfer of laminar boundary layers developed over inclined, isothermal plate and have confirmed that the local Nusselt numbers can be correlated with the above Rayleigh numbers, $R a_{x \theta}$. Thus, it is considered that the heat transfer characteristics of the laminar flows have been clarified to a considerable extent.

Meanwhile, for the heat transfer of turbulent flows,
Black and Norris ${ }^{[3]}$ have measured the local heat transfer coefficients from the isothermal plate at inclination angles $\theta$ $=10,20,30$ and $45^{\circ}$. They reported that the local Nusselt numbers can be correlated well with the Rayleigh numbers, $R a_{x}\left(=g \beta \Delta T x^{3} / \alpha v\right)$, where the acceleration in $R a_{x}$ was taken as the normal gravity, $g$. They also showed that the correlation for the inclined plates coincides with the conventional correlation for the turbulent flows, indicating that the Nusselt numbers are independent of the inclination angles. Meanwhile, Al-Arabi and $\mathrm{Sakr}^{[4]}$ have measured the heat transfer coefficients of the turbulent flows developed over the isothermal plate by varying the inclination angles, $\theta=10-90^{\circ}$ from horizontal. However, they have reported that the local Nusselt numbers depend on the inclination angles, and the numbers increase monotonously with decreasing inclination angles. Thus, there are still some arguments concerning with the turbulent heat transfer.

Meanwhile, turbulent transition mechanisms over the inclined plates have also attracted considerable interests of the previous workers. The visualization study by Sparrow and Husar ${ }^{[5]}$ have first revealed that longitudinal vortices of which axes are parallel to the flow direction appear over the plate in advance of the turbulent transition. Chen and $\mathrm{Kim}^{[6]}$ have also carried out the visualizations of air flows over the inclined plate using smoke. The onset and the spanwise pitches of the vortices were measured quantitatively in their study.

Although these heat-transfer and flow visualization studies provide basic insight into the flow and heat transfer characteristics of the natural convection over inclined plates, we can point out some faults in the previous experiments. The one of such fault is that the main concerns of the previous workers have been directed to either the heat transfer characteristics or the turbulent transition 
mechanisms, so that the measurements have been conducted almost independently with each other. This, however, hinders us in discussing further the relation between the flow and heat transfer of the natural convection, in particular, when a turbulent transition undergoes over the plate. Another fault is that the previous investigations have been carried out in a narrow range of inclination angles. This does not allow us to draw a complete picture of the heat transfer and the turbulent transition over plates inclined from horizontal to vertical.

Taking these into account, intensive visualization experiments using smoke have been first carried out in the present study. Then, the measurements of the local heat transfer coefficients were conducted in the laminar, transitional and turbulent flow regimes. The measurements cover the full range of inclination angles. The present results will provide the comprehensive information on the relation between the flow fields over the plate and the local heat transfer characteristics.

\section{EXPERIMENTALAPPARATUS AND MEASUREMENT}

In the present experiments, air at room temperature was utilized as a test fluid, and a test plate heated with constant heat flux was also adopted. A schematic illustration of the present experimental apparatus is shown in Fig.1. The test plate was fabricated with an acrylic resin base plate $3 \mathrm{~mm}$ thick and stainless-steel foil heaters $30 \mu \mathrm{m}$ thick. The heaters were glued on the surface of the base plate. A constant heat flux condition was accomplished by supplying $\mathrm{AC}$ power to the heaters. The test plate of $1800 \mathrm{~mm}$-long and $700 \mathrm{~mm}$-wide was utilized for the experiments. A $50 \mathrm{~mm}$ thick Styrofoam plate was glued on the back of the test plate to inhibit a conduction heat loss. Also, side plates of $250 \mathrm{~mm}$-high were attached on the both sides of the test plate to inhibit the side flows and to realize a two-dimensional flow field over the plate. The test plate was fixed on a stage with arbitrary angles from horizontal $\left(0^{\circ}\right)$ to vertical $\left(90^{\circ}\right)$.

The above apparatus was placed on the floor of a small experimental room of $1.2 \mathrm{~m} \times 2.4 \mathrm{~m}$ cross-sectional area and $3 \mathrm{~m}$-high. The floor and the ceiling of the room were fabricated with punched metal sheets. These metal sheets

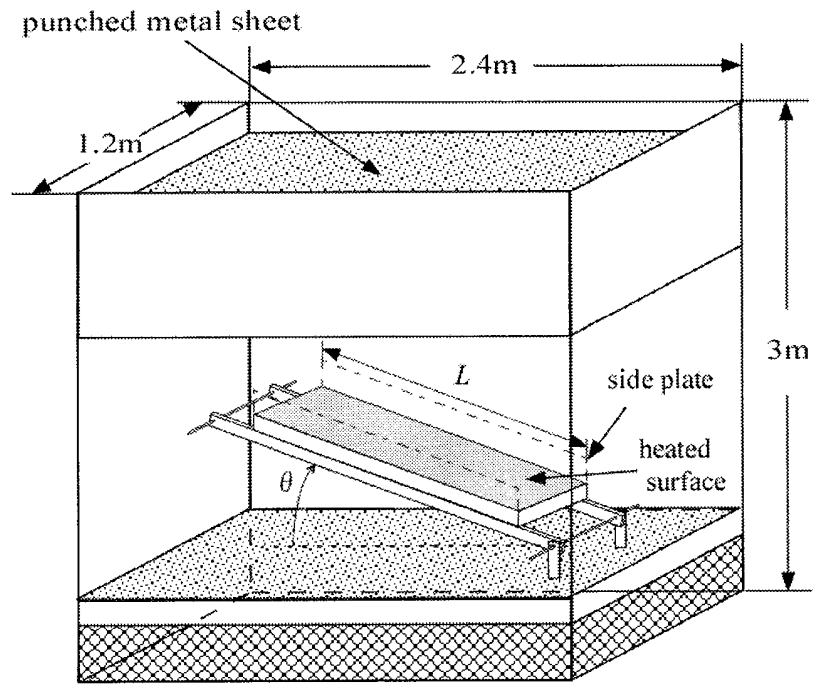

Fig.1 Experimental Apparatus enabled to introduce a fresh air of small turbulences from the floor and to exhaust a high-temperature plume smoothly from the ceiling.

For the measurements of the local heat transfer coefficients, Chromel-Alumel thermocouples of $100 \mu \mathrm{m}$ diameter were spot-welded on the back of the heaters along the centerline of the test plate. These thermocouples measure the local surface temperature of the plate, $T_{w x}$, in the streamwise direction. The temperature of the ambient fluid, $T_{\infty}$, was also measured with the thermocouples, which were placed $50 \mathrm{~mm}$ apart from the bottom edge of the test plate and in the same horizontal plane. Based on the temperature difference between the surface and ambient temperatures, $T_{x}, T_{\infty}$, and also on a convective heat flux, $q_{w}$, local heat transfer coefficients, $h_{x}$, were defined and calculated as follows:

$$
h_{x}=q_{w} /\left(T_{w x}-T_{\infty}\right)
$$

In the experiments with air, heat losses by radiation from the surface and by conduction through the base plate could not be neglected against the heat transfer by a natural convection. Thus, we estimated the radiation heat loss as:

$$
q_{\text {rad }}=\sigma \varepsilon\left(T_{w x}{ }^{4}-T_{\infty}{ }^{4}\right)
$$

Where, $q_{\text {rad }}$ stands for a heat flux by radiation from the surface of temperatures, $T_{w x}$, to the environmental air of temperature, $T_{\infty}$. The notations $\sigma$ and $\varepsilon$ show the StefanBoltzmann's constant and the emissivity. We adopted $\varepsilon=$ 0.16 as the emissivity of the stainless steel heaters. While, the heat flux lost by conduction, $q_{\text {cond }}$, was estimated by assuming a one-dimensional heat conduction through the foam-polystyrene plate. Then, the convective heat flux, $q_{w}$, was calculated by subtracting the above heat fluxes from the Joule heat flux, $q_{j}$, deposited on the heater as: $q_{w}=q_{j}$ $\left(q_{\text {cond }}+q_{\text {rad }}\right)$. In the experiments, the heat fluxes lost by radiation and by conduction were estimated as: $q_{\mathrm{rad}}$ $=16-21 \%$ and $q_{c o n d}=11-16 \%$ of the Joule heat flux, $q_{j}$. The experiments covered the ranges of the modified Rayleigh numbers up to $R a_{L}{ }^{*}=10^{13}$, where the numbers were based on the length of the plate as $R a_{L}{ }^{*}\left(=g \beta q_{w} L^{4} / \lambda \alpha v\right)$. Thermo-physical properties of non-dimensional parameters mentioned in the below were estimated at the film temperatures as: $T_{f}=\left(T_{w}+T_{\infty}\right) / 2$, while the volume expansion coefficient $\beta$ of the air was estimated at the ambient temperature as: $\beta=1 / T_{\infty}$.

\section{RESULTS AND DISCUSSION}

\subsection{Visualization of Flow Fields}

In order to obtain comprehensive information on the flows over the inclined plate, we first carried out the flow visualizations at first. The representative results are shown in Fig. 2 and Fig.3. For the visualizations, the smoke was issued slowly from a slit flush-mounted on the bottom edge of the plate. The photos in those figures were taken directly from the front of the plate. Fig.2 represents the flow fields over the plate of inclination angles $\theta=0-60^{\circ}$. As is obvious from the figures, the smoke issued from the slit first flows along the plate first, and then, concentrates to become a filament at certain distance from the leading edge. The smoke filament, then, detaches from the surface and 
becomes distorted toward downstream. Finally, a fully turbulent state is accomplished at far downstream from the leading edge. We should note here that the flow field over the horizontal plate become symmetrical about the horizontal centerline of Fig.2(a), because the flows from the both sides of the plate are blocked with side plates.

Similar experiments as above have been conducted by Sparrow and Husar ${ }^{[5]}$ and Lloyd and Sparrow ${ }^{[7]}$, where the natural convective flows of water over inclined, isothermal plate were visualized using an electrochemical dye marker method. They reported that the dye filaments appear first over the plate, and then, the filaments detach from the surface as were observed by the present experiments. They also revealed that the formation and the detachment of dye filaments are caused by the longitudinal vortices, with axes parallel to the flow direction. That is, the dye filaments appear first at the legs of counter- rotating pair vortices, and, then, detaches with the growing of these vortices toward downstream.

Meanwhile, Figures 3(a) and 3(b) show the flow fields over the plate of inclination angles $\theta=75^{\circ}$ and $90^{\circ}$. As is obvious from Fig.3(b), the flow field over the vertical plate $\left(\theta=90^{\circ}\right)$ are altered substantially from those over the inclined plate shown in Fig.2. We will see long waves traverse to the flow direction in Fig.3(b). The figure also shows that the waves are gradually distorted toward downstream, and a fully turbulent state is achieved far downstream of the plate. The above transition phenomena are generally called as the Tollmien-Schlichting's wave instability. Careful observation of Fig.3(b) also finds the waves over the plate of $\theta=75^{\circ}$ together with the filaments. Summarizing the above results, two kind of instability are found to occur over the inclined plate. Those are the vortex and the wave instabilities that appear over the plates with smaller and larger inclination angles. These instabilities were found first in the visualization experiments by Lloyd and Sparrow ${ }^{[7]}$. They reported that the former and latter instability occur when $\theta<73^{\circ}$ and $\theta>76^{\circ}$, respectively. Their result was obtained in experiments with isothermal plate and water, while the present experiments have been conducted with constant heat flux plate and air. The differences in heating condition and test fluid may affect the above instabilities. Thus, we next examined the criteria for these instabilities by varying the inclination angles with

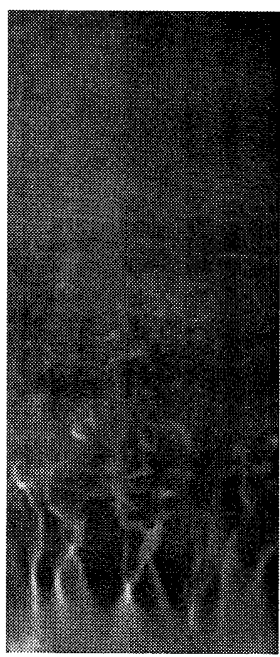

(a) $\theta=0^{\circ}$

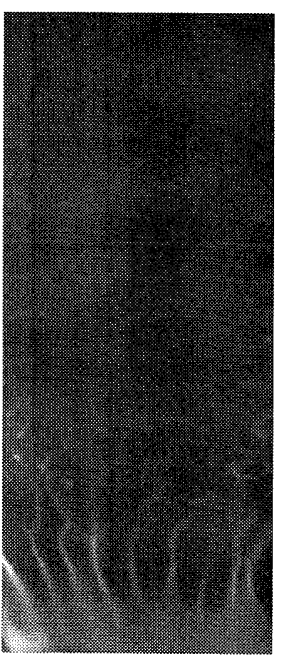

(b) $\theta=15^{\circ}$

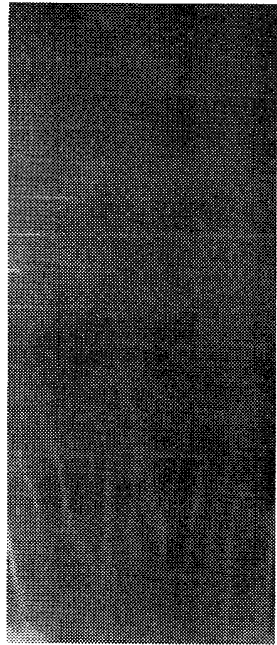

(c) $\theta=30^{\circ}$ an interval of $1^{\circ}$ from $70^{\circ}$ to $80^{\circ}$. The results showed that the vortex and wave instability occurs when $\theta<72^{\circ}$ and $\theta>78^{\circ}$, while both instabilities occur in between them, $72^{\circ}<\theta<78^{\circ}$. The above criteria agree fairly well with those of Lloyd and Sparrrow ${ }^{[7]}$. Thus, it is concluded that the heating condition and test fluid have no serious influence on these instabilities, and that they are determined solely by the inclination angles.

\subsection{Onset and Detachment of Longitudinal Vortex}

From the visualization experiments mentioned above, it was found that the generation and detachment of the longitudinal vortices exert serious influences on the turbulent transition. Thus, for further investigations on the turbulent transition, quantitative data on the onset and detachment of the vortices and on their spanwise pitches are indispensable. Thus, we have next measured these quantities from the flow visualization experiments conducted with various inclination angles and surface heat fluxes.

In the course of the measurements, we observed that the onsets and the detachment points move irregularly with

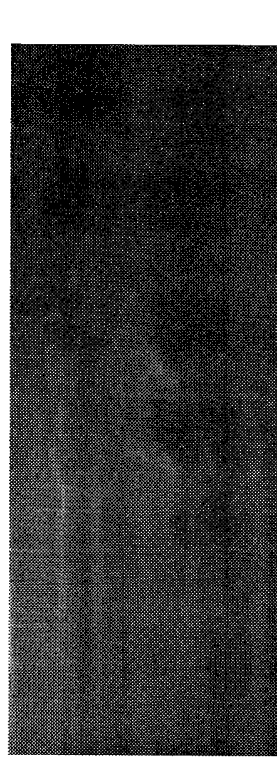

(a) $\theta=75^{\circ}$

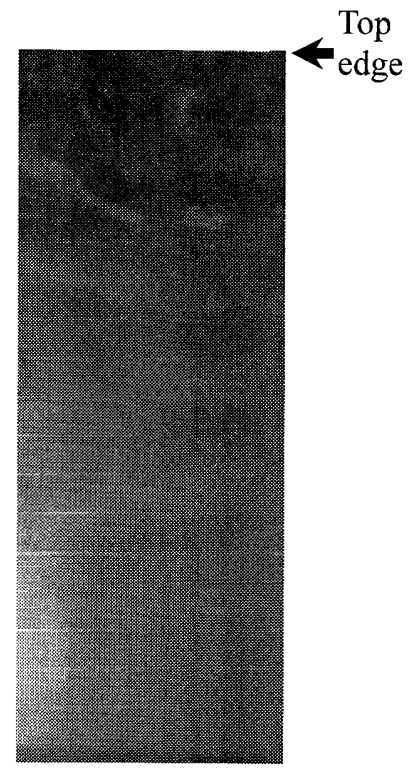

(b) $\theta=90^{\circ}$
Fig. 3 Visualized flow fields $\left(\theta=75,90^{\circ}\right)$

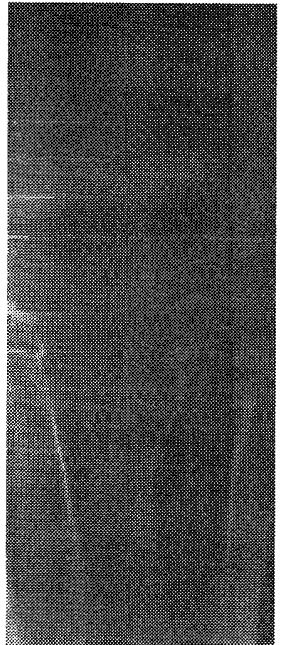

(d) $\theta=45^{\circ}$

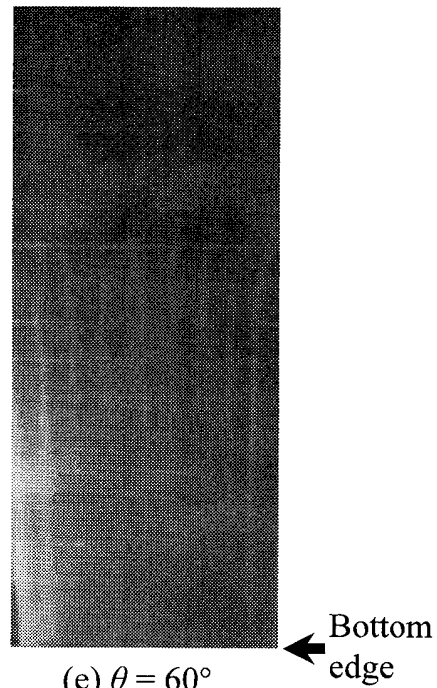

Fig. 2 Visualized flow fields $\left(\theta=0-60^{\circ}\right)$ 
a period of several to 10 seconds. Thus, we defined the onsets and detachment points with their time-averaged location. The results on the onsets and separation points are shown in Fig.4. In the figure, the distances from bottom edge to the onset and to the detachment points, $x_{1}$ and $x_{2}$, are normalized with the modified Rayleigh numbers as $\left.R a_{x 1}{ }^{*}{ }^{\prime}=g \beta q_{w} x_{1}{ }^{4} / \lambda \alpha \nu\right)$ and $R a_{x 2}{ }^{*}\left(=g \beta q_{w} x_{2}{ }^{4} / \lambda \alpha \nu\right)$, respectively, and the numbers are plotted in term of the inclination angles, $\theta$. Meanwhile, the present authors ${ }^{[8]}$ have carried out the same measurements of the above numbers for the case of water. The data are also plotted in Fig. 4 for comparison. It is obvious from the figure that the Rayleigh numbers, $R a_{x 1}{ }^{*}$ and $R a_{x 2}{ }^{*}$, corresponding to the onsets and the detachment points increase monotonically with the inclined angles. The figure also depicts that $R a_{x 1}{ }^{*}$ and $R a_{x 2}{ }^{*}$ gradually depart from each other with increasing the angles. Moreover, the results for air show higher Rayleigh numbers, $R a_{x 1}{ }^{*}$, than those for water, while the Rayleigh numbers for the detachment points, $R a_{x 2}{ }^{*}$, are almost identical between air and water.

We have next measured the spanwise pitches of the longitudinal vortices. The results showed that the pitches $p$ ranged from 22 to $34 \mathrm{~mm}$ and they became smaller with the heat fluxes and larger with the inclination angles. The present authors ${ }^{[8]}$ have already conducted similar measurements on the pitches with water, and the pitches ranged from 6 to $11 \mathrm{~mm}$. According to their results, the pitches normalized with the characteristic length, $l$ $\left(=\lambda \alpha v / g \beta q_{w}\right)^{1 / 4}$, show constant values regardless of surface heat fluxes. Making use of the result, present pitches for air were also normalized with the above length scales. The result is presented in Fig.5, where normalized pitches $(p / l)$ are plotted with the inclination angles. The figure shows that the dimensionless pitches increase slightly with the inclination angles, while the values are almost the same between the air and water.

\subsection{Local Heat Transfer Characteristics}

In light of the above results on the flow visualizations, we subsequently carried out the measurements of the local heat transfer coefficients using thermocouples. The representative results are shown in Fig.6, where the local heat transfer coefficients, $h_{x}$, of the inclined plate of $\theta=30$ and $60^{\circ}$ are plotted with the streamwise distance, $x$, from the bottom edge of the plate. It is apparent from the both figures 6(a) and 6(b) that the highest coefficients are realized at the leading edge of the plate, and the coefficients decrease rapidly with $x$ and reach minima. Then, they turn to increase and show maxima. In the downstream region of the maxima, the coefficients decrease gradually with $x$. Moreover, the coefficients increase monotonously with the heat flux for all surfaces, while the location of minimum and maximum coefficient shifts to the leading edge with the heat flux. We denoted the onsets and detachment points of the longitudinal vortices with the arrows $\uparrow$ and $\downarrow$ in the figures. The figures depict that the onsets and the detachment points are located just upstream and downstream of the minimum coefficients.

Based on these heat transfer coefficients, we next calculated the local Nusselt numbers, $N u_{x}\left(=h_{x} \cdot x / \lambda\right)$, and the numbers are plotted in terms of the local modified Rayleigh numbers, $R a_{x}^{*}\left(=g \beta q_{w} x^{4} / \lambda \alpha v\right)$ as shown in Fig.7. The Rayleigh numbers corresponding to the onsets, $R a_{x 1}{ }^{*}$, and

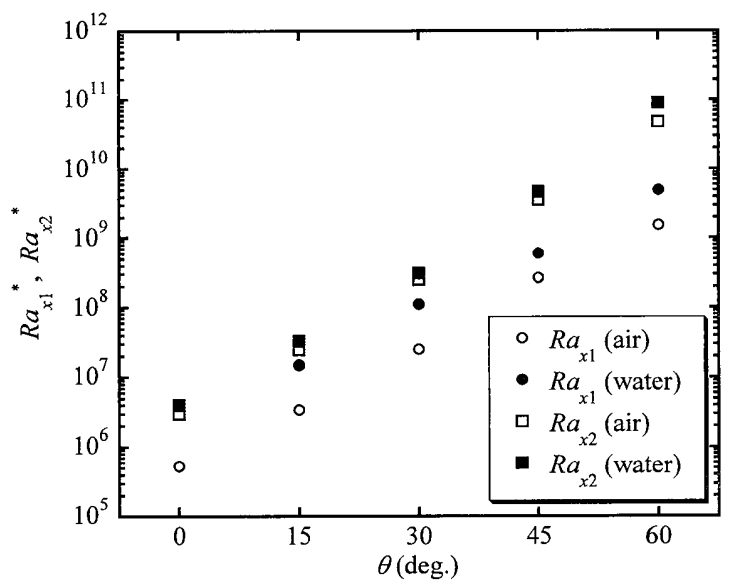

Fig.4 Rayleigh numbers for the onset and detachment of longitudinal vortex

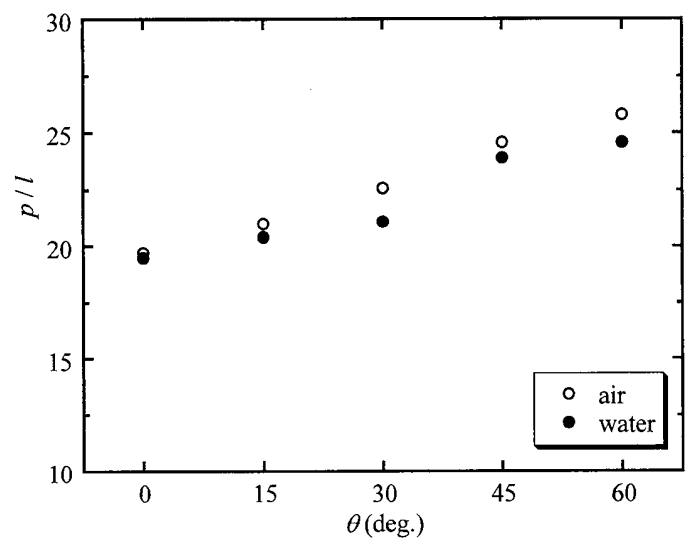

Fig.5 Spanwise pitches of longitudinal vortex

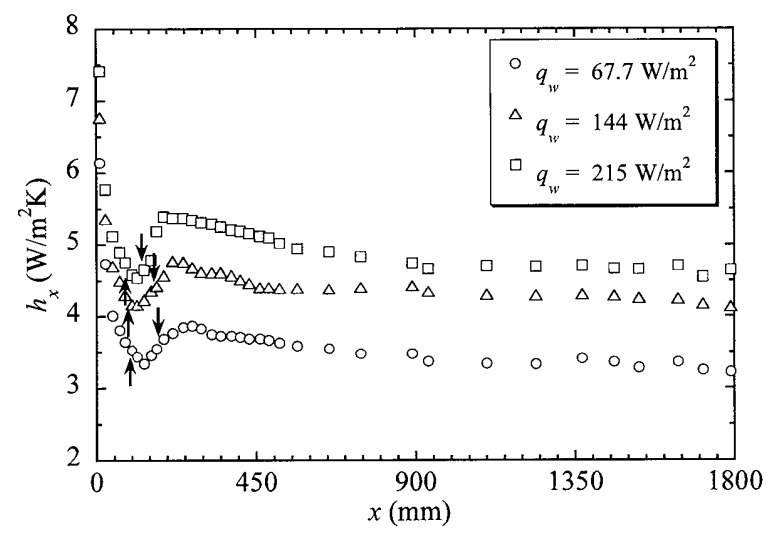

(a) $\theta=30^{\circ}$

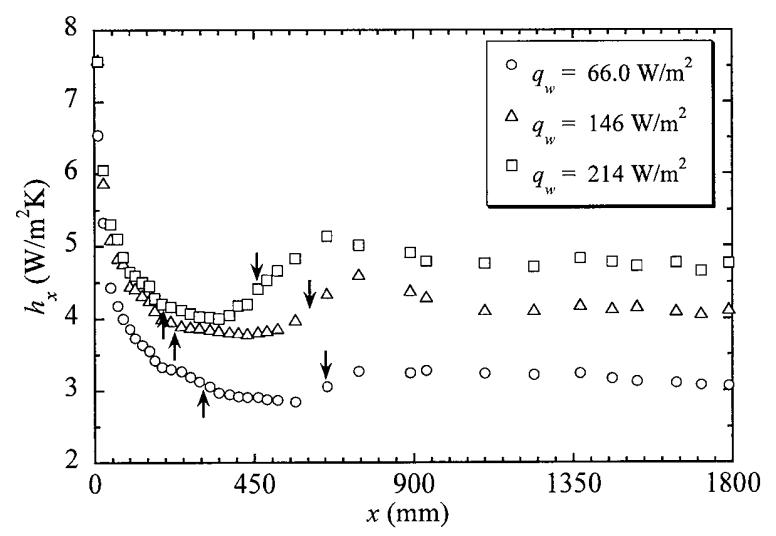

(b) $\theta=60^{\circ}$

Fig. 6 Local heat transfer coefficients from inclined plate 
the detachment points, $R{a_{x 2}}^{*}$, are also denoted with arrows $\uparrow$ and $\downarrow$ in the figure. Although the present measurements have been performed for all inclination angles from horizontal to vertical with increments of $15^{\circ}$, the results only for $\theta=0,30,60,90^{\circ}$ are plotted to avoid the confusion of data. Besides, accounting the symmetrical flow configuration of the horizontal plate, the data from the edge to the center of the horizontal plate are plotted in the figure. Therefore the maximum Rayleigh number remained small by one digit compared with those of the inclined plates. The figure depicts that the local Nusselt numbers increase linearly with the Rayleigh numbers for all inclination angles. One will also see that the Nusselt numbers at each inclination angles show different linear variations against the Rayleigh numbers. The one is for the low Rayleigh numbers less than $R a_{x 1}{ }^{*}$. Another is for the high Rayleigh numbers larger than $R a_{x 2}{ }^{*}$. These two represent the correlation for the laminar and turbulent heat transfer. A careful observation on the former plots will find that their gradients gradually become steep with the inclination angles. As was mentioned in Section 1, the local Nusselt numbers for the laminar flows can be correlated with the local modified Rayleigh numbers, $R a_{x \theta}{ }^{*}\left(=g \sin \theta \cdot \beta q_{w} x^{4} / \lambda\right.$ $\alpha v$ ), of which the gravitational acceleration is $g \sin \theta$. Accounting this, the same arrangement was carried out for the present data. The result is given in Fig. 8 together with previous result for water obtained by the present authors ${ }^{[8]}$, which is shown with the dotted line in the figure. It is apparent that the all of the present air data except for $\theta=0^{\circ}$ gather around the single line expressed as:

$$
N u_{x}=0.53 R a_{x}{ }^{* 1 / 5}
$$

We have found that Eq. (3) coincides well with the analytical correlation proposed by Chen and colleagues ${ }^{[2]}$, who have conducted the similarity analysis on the laminar boundary layer flows over the inclined plate and have obtained the following correlation.

$$
N u_{x}=C(P r) \cdot R a_{x}{ }^{*} 1 / 5
$$

Where $C(P r)$ is a function of Prandtl number and is expressed as:

$$
C(\operatorname{Pr})=[\operatorname{Pr} /(4+9 \sqrt{\operatorname{Pr}}+10 \cdot \operatorname{Pr})]^{1 / 5}
$$

Substituting the present Prandtl number of air, $\mathrm{Pr}=$ 0.72 , into their correlation yields:

$$
N u_{x}=0.52 R a_{x \theta}{ }^{* 1 / 5}
$$

The above result also confirms the accuracy of the present measurements. Moreover, the present air data show smaller Nusselt numbers by $10 \%$ compared to those for water. This is attributable to the Prandtl number effect on the Nusselt numbers.

We next discuss the turbulent heat transfer, which is our main concern. In Figure 9, all of the present air data and the previous water data by the authors ${ }^{[8]}$ are plotted on the same $N u_{x}-R a_{x}{ }^{*}$ plane, where the gravitational acceleration $g$ is adopted for the local Rayleigh numbers as: $R a_{x}{ }^{*}=g \beta$ $q_{w} x^{4} / \lambda \alpha v$. It is seen that the present air data give almost

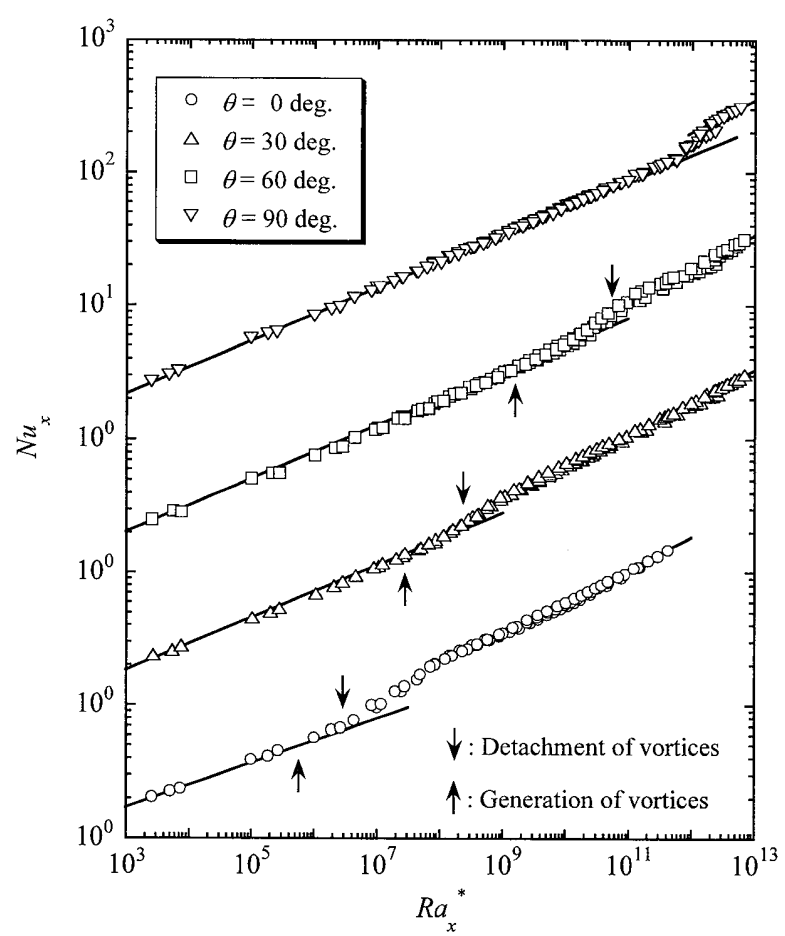

Fig.7 Local Nusselt numbers of inclined plate

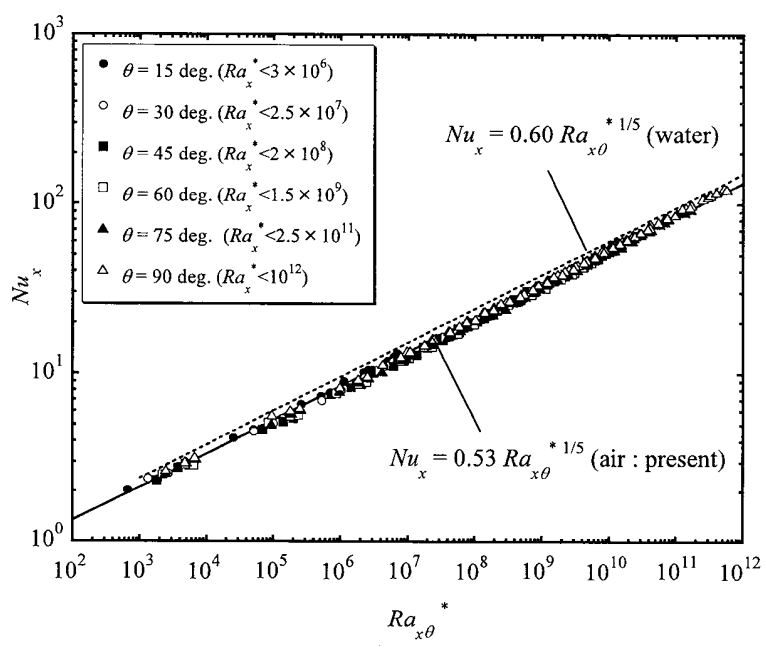

Fig.8 Local Nusselt Numbers of laminar flow

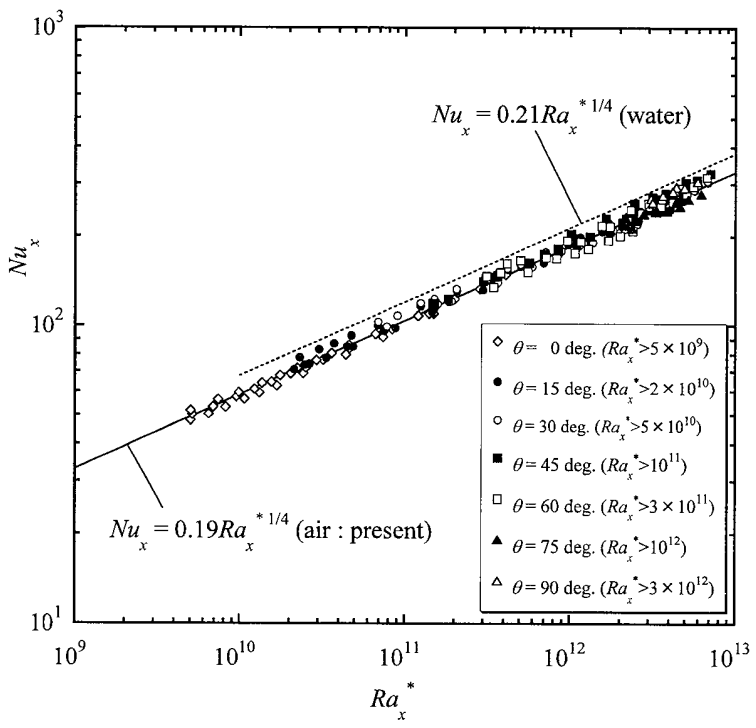

Fig.9 Local Nusselt Numbers of turbulent flow 
identical Nusselt numbers for all inclination angles from horizontal to vertical, and the correlation between the Nusselt and Rayleigh numbers can be expressed with the following equation:

$$
N u_{x}=0.19 R a_{x}^{* 1 / 4}
$$

The above correlation indicates that the Nusselt numbers for the turbulent flows over horizontal, inclined and vertical plates become identical, and that are determined with the local Rayleigh numbers. Besides, present air data show $10 \%$ smaller Nusselt numbers compared to the previous water data. Although it is well recognized that the turbulent Nusselt numbers from the vertical plate depend on the Prandtl numbers, none has confirmed that the same result is obtained for the inclined plate. Thus, the present results seem worthwhile and will provide useful information for the future analyses.

\section{CONCLUSIONS}

The fluid flow and heat transfer of natural convection adjacent to inclined plates were investigated experimentally in the present study. The flow field over the plate was visualized with smoke and the local heat transfer coefficients from the plate were also measured with thermocouples. Then, we discussed the relations between the flow field and heat transfer to obtain a comprehensive knowledge on the natural convection over inclined, heated plate. In the experiments, air at room temperature was used as a test fluid. The test plate was heated with constant heat fluxes and the inclination angles were varied systematically from horizontal to vertical. The test plate of $1800 \mathrm{~mm}$-long enabled the experiments with maximum Rayleigh numbers of $10^{13}$. The following results were obtained from the above experiments.

(1) The flow visualization experiments revealed that the following two instabilities appear over the inclined plate. The first is the vortex instability that occurs over the plate of $\theta<72^{\circ}$, where the longitudinal vortices first appear over the plate, and then, detach from the surface and are distorted to become a fully turbulent state. The present results showed that the onsets and the detachment points of the above vortices shift toward downstream and they depart from each other with increasing inclination angles from horizontal. The spanwise pitches of the vortices also show gradual increase with the inclination angles. The second is the wave instability that occurs over the plate of $\theta>78^{\circ}$, where the long waves parallel to the flow direction appear first over the plate, and then, are distorted toward downstream and finally, a fully turbulent state is realized. We also found that the above two instabilities take place over the plate of the intermediate angles, $72^{\circ}<\theta<78^{\circ}$.

(2) The local heat transfer coefficients were also measured with the thermocouples under various inclination angles and surface heat fluxes. The results showed that the local Nusselt numbers in the laminar region are correlated with the modified local Rayleigh numbers, of which the gravitational acceleration is $g \sin \theta$. While, the local Nusselt numbers for the turbulent flows can be correlated with the conventional Rayleigh numbers, and the numbers become independent of inclination angles. Based on these data, empirical correlations between the local Nusselt numbers and the Rayleigh numbers were proposed for both laminar and turbulent flows. The correlations cover the ranges of the modified Rayleigh numbers, $R a_{x}{ }^{*}=10^{3}$ to $10^{13}$, and the inclination angles, $0^{\circ}<\theta<90^{\circ}$.

\section{REFERENCES}

1. Hassan, K. L. and Mohamed, S.A., Natural Convection from Isothermal Flat Surfaces, Int. J. Heat Mass Transf. Vol.13, (1970), pp.1873-1886.

2. Chen, T. S., Tien, H. C. and Armaly, B.F., Natural Convection on Horizontal, Inclined, and Vertical Plates with Variable Surface Temperature or Heat Flux", Int. J. Heat Mass Transf., Vol.29 (1986), pp.1465-1478.

3. Black, W. Z. and Norris, J. K., The Thermal Structure of Free Convection Turbulence from Inclined Isothermal Surfaces and Its Influence on Heat Transfer, Int. J. Heat Mass Transf., Vol.18 (1975), pp.43-50.

4. Al-Arabi, M. and Sakr, B., Natural Convection Heat Transfer from Inclined Isothermal Plates, Int. J. Heat Mass Transf., Vol.31 (1988), pp.559-566.

5. Sparrow, E. M. and Husar, R. B., "Longitudinal Vortices in Natural Convection Flow on Inclined Surfaces", J. Fluid Mech., Vol. 37 (1969), pp. 251-255.

6. Lloyd, J. R., Sparrow, E. M. and Eckert, E. R. G., "Laminar, Transition and Turbulent Natural Convection Adjacent to Inclined and Vertical Surfaces", Int. J. Heat Mass Transf. Vol.15, (1972), pp. 457-473.

7. Cheng, K.C. and Kim, Y.W., Flow Visualization Studies on Vortex Instability of Natural Convective Flow over Horizontal and Slightly Inclined Constant-Temperature Plates, Trans. ASME, J. Heat Transf., Vol.110 (1988), pp.608-615

8. Kimura, F. , Kitamura, K., Yamaguchi, M. and Asami, T., Fluid Flow and Heat Transfer of Natural Convection Adjacent to Upward-Facing Inclined, Heated Plates, Transactions of the Japan Society of Mechanical Engineers, Series B, Vol. 66, No. 645 (2000), pp.1453-1461.(in Japanese) 